Slavica

bruxellensia

\section{Slavica bruxellensia}

Revue polyphonique de littérature, culture et histoire

slaves

$10 \mid 2014$

Espace slave, espace germanique

\title{
Igor Stravinskij. Confidences sur la musique. Propos recueillis (1912-1939)
}

\author{
Izabela Jach
}

\section{OpenEdition}

Journals

Édition électronique

URL : http://journals.openedition.org/slavica/1630

DOI : 10.4000/slavica.1630

ISSN : 2034-6395

\section{Éditeur}

Université libre de Bruxelles - ULB

\section{Référence électronique}

Izabela Jach, « Igor Stravinskij. Confidences sur la musique. Propos recueillis (1912-1939) », Slavica bruxellensia [En ligne], 10 | 2014, mis en ligne le 15 avril 2014, consulté le 22 septembre 2020. URL: http://journals.openedition.org/slavica/1630 ; DOI : https://doi.org/10.4000/slavica.1630

Ce document a été généré automatiquement le 22 septembre 2020.

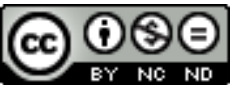

Les contenus de Slavica bruxellensia sont mis à disposition selon les termes de la Licence Creative Commons Attribution - Pas d'Utilisation Commerciale - Pas de Modification 3.0 France. 


\section{Igor Stravinskij. Confidences sur la musique. Propos recueillis (1912-1939)}

Izabela Jach

\section{RÉFÉRENCE}

Igor Stravinskij. Confidences sur la musique. Propos recueillis (1912-1939). Textes et entretiens choisis, édités et annotés par Valérie Dufour,Actes Sud, Arles, 2013, 404 p. 
1 Qui est Igor Stravinskijj ? Pour certains, c'est un génie, pour d'autres, un fou ou un démon. Sans aucun doute, il est une icône de l'époque contemporaine, considéré comme l'un des compositeurs les plus influents $\mathrm{du} \mathrm{xx}^{\mathrm{e}}$ siècle. Le génie avec lequel il est intervenu dans le monde de la musique a fait que sa bibliographie est riche. L'ouvrage de Valérie Dufour est néanmoins unique parce que dans ces Confidences sur la musique, c'est le maîtremême Stravinskijj qui prend la parole. C'est un vrai régal intellectuel pour les

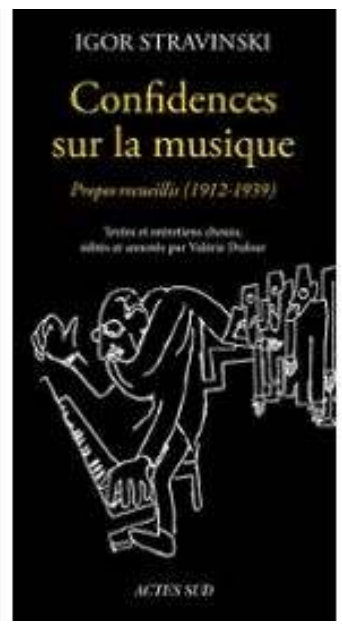
amateurs comme pour les mélomanes chevronnés. Il convient de souligner que Dufour a déjà publié divers textes sur Stravinskijj et sur la vie musicale des $\mathrm{XIX}^{\mathrm{e}}$ et $\mathrm{XX}^{\mathrm{e}}$ siècles.

2 L'ouvrage contient des écrits que l'artiste a publiés dans la presse avant 1940 et un choix d'entretiens qu'il a accordés à la même époque. Dans ses lettres, le musicien nous parle de modernité, de technologie, progrès, politique et critique, de ses inspirations et fascinations pour lui-même ainsi que de sa compréhension du monde et de l'art. Les Confidences sur la musique jettent une nouvelle lumière sur la personnalité créative de Stravinskij. La chercheuse nous propose une rencontre intime avec le musicien qui parle de son enfance dans l'opéra, de son père, ou encore de sa rencontre avec Nikolaj Rimskij-Korsakov auquel il a avoué qu'il voulait être compositeur. Dufour a créé une œuvre grâce à laquelle nous pouvons composer un portrait complexe de Stravinskij en tant qu'artiste et en tant qu'être humain. Elle introduit le lecteur dans le monde intérieur de l'artiste. Dans ce monde surréaliste et spirituel, l'auteure est comme un guide qui a judicieusement choisi les textes et les entretiens, lesquels tiennent compte de l'évolution artistique de Stravinskij, de sorte que le lecteur ne soit pas perdu dans le labyrinthe des pensées du génie.

3 Dans la première partie de l'ouvrage, les lettres sont avant tout le reflet de ses chefsd'œuvre, comme par exemple Le Sacre du printemps ou Perséphone. Le compositeur explique également ce qu'il a vraiment voulu exprimer dans ses ballets, il parle de ses inspirations, notamment de Puškin et Čajkovskij, il évoque l'histoire de la Russie ou encore sa candidature à l'Institut en France. Tout cela est accompagné d'une vaste réflexionsur la politique et la musique. La plume du compositeur n'a pas de grande valeur littéraire et ses observations confirment que Stravinskij n'est pas un écrivain mais plutôt un penseur - philosophe qui choisit ses mots avec soin. L'image de l'artiste dans ces fragments n'est pas facile à définir. Nous ne pouvons savoir si Stravinskijest un moderniste, un révolutionnaire ou encore un fou qui ne respecte aucune tradition.

4 La deuxième partie des Confidences sur la musique se compose d'entretiens, mais Dufour souligne que les entretiens dans la presse doivent toujours être lus avec prudence et attention parce que nous ne pouvons pas toujours vérifier la qualité de la transcription du journaliste. Stravinskij a parfois avoué qu'il ne se reconnaissait pas dans les interviews présentées. Mais pour nous lecteurs, les interviews journalistiques ont une grande valeur puisque nous y trouvons les pensées spontanées de Stravinskij. Il parle de sa vie spirituelle, des événements importants des années vingt. Nous découvrons la 
fascination de l'artiste pour l'Amérique et pour Rome ou encore son opinion sur le jazz, sur la musique et le cinéma, sur la radio et les enregistrements à la radio. Le compositeur se prononce à la fin sur ses méthodes et ses buts dans le domaine de la musique. L'artiste est présenté, si l'on considère que tous les entretiens ont été autorisés, par ses propres paroles et son portrait est complété par un grand nombre de citations.

5 En somme, Dufour nous propose un voyage extraordinaire à travers le monde intérieur d'une personnalité fascinante et controversée, laquelle n'est pas complètement définie à ce jour. L'auteure laisse le soin au lecteur de trouver sa propre réponse à la question de savoir qui est Stravinskij et quel est son rôle dans l'art contemporain. Elle nous invite à nous interroger sur la signification même du génie et sur la frontière entre la folie et le génie. Ce qui rend l'ouvrage aussi intéressant et compréhensible pour les connaisseurs de Stravinskij que pour un novice qui ne le connaît pas ou seulement juste un peu mais qui, une fois la lecture entamée ne peut s'arrêter de lire avec fascination. 